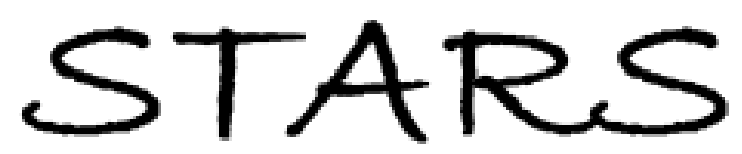

University of Central Florida

STARS

Faculty Bibliography 2010s

Faculty Bibliography

$1-1-2014$

\title{
Study of filamentation threshold in zinc selenide
}

\author{
Magali Durand \\ University of Central Florida \\ Aurélien Houard \\ Khan Lim \\ University of Central Florida \\ Anne Durécu \\ Olivier Vasseur
}

See next page for additional authors

Find similar works at: https://stars.library.ucf.edu/facultybib2010

University of Central Florida Libraries http://library.ucf.edu

This Article is brought to you for free and open access by the Faculty Bibliography at STARS. It has been accepted for inclusion in Faculty Bibliography 2010 s by an authorized administrator of STARS. For more information, please contact STARS@ucf.edu.

\section{Recommended Citation}

Durand, Magali; Houard, Aurélien; Lim, Khan; Durécu, Anne; Vasseur, Olivier; and Richardson, Martin, "Study of filamentation threshold in zinc selenide" (2014). Faculty Bibliography 2010s. 5282.

https://stars.library.ucf.edu/facultybib2010/5282

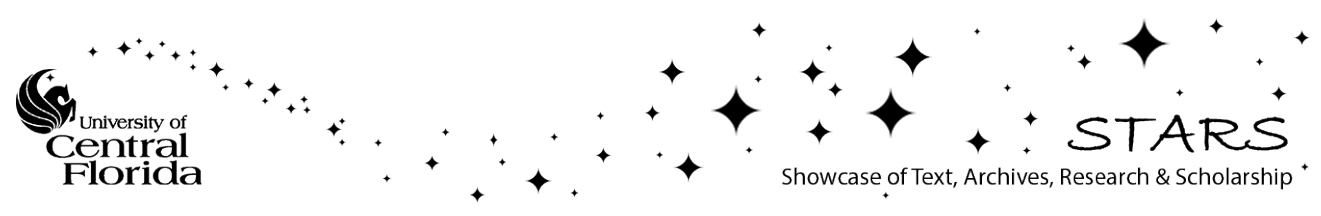




\section{Authors}

Magali Durand, Aurélien Houard, Khan Lim, Anne Durécu, Olivier Vasseur, and Martin Richardson 


\title{
Study of filamentation threshold in zinc selenide
}

\author{
Magali Durand, ${ }^{1, *}$ Aurélien Houard, ${ }^{2}$ Khan Lim, ${ }^{1}$ Anne Durécu, ${ }^{3}$ Olivier Vasseur, ${ }^{3}$ \\ and Martin Richardson ${ }^{1}$ \\ ${ }^{1}$ Townes Laser Institute, CREOL - The College of Optics and Photonics, University of Central Florida, Orlando, \\ Florida 32816, USA \\ ${ }^{2}$ Laboratoire d'Optique Appliquée, ENSTA ParisTech - Ecole Polytechnique, CNRS, Palaiseau, F-91762, France \\ ${ }^{3}$ ONERA, Chemin de la Hunière, 91761 Palaiseau, France \\ "Magali.durand@creol.ucf.edu
}

\begin{abstract}
The possibility of creating filaments with laser wavelengths ranging from $800 \mathrm{~nm}$ to $2.4 \mu \mathrm{m}$ was investigated using an OPA laser system. Zinc Selenide's (ZnSe) unique characteristics - small band gap $E_{\text {gZnSe }}=2.67 \mathrm{eV}$ and positive dispersion for this wavelength range - are well suited for filamentation study where multi-photon absorption can be achieved with two to six photons.
\end{abstract}

(C)2014 Optical Society of America

OCIS codes: (320.2250) Femtosecond phenomena; (320.7110) Ultrafast nonlinear optics; (190.4180) Multiphoton processes.

\section{References and links}

1. J. H. Marburger, "Self-focusing: Theory," Prog. Quantum Electron. 4, 35-110 (1975).

2. M. Sheik-Bahae, D. J. Hagan, and E. W. Van Stryland, "Dispersion and band-gap scaling of the electronic Kerr effect in solids associated with two-photon absorption," Phys. Rev. Lett. 65(1), 96-99 (1990).

3. A. Couairon and A. Mysyrowicz, "Femtosecond filamentation in transparent media," Phys. Rep. 441(2-4), 47189 (2007).

4. S. L. Chin, S. A. Hosseini, W. Liu, Q. Luo, F. Théberge, N. Aközbek, A. Becker, V. P. Kandidov, O. G. Kosareva, and H. Schroeder, "The propagation of powerful femtosecond laser pulses in optical media: physics, applications, and new challenges," Can. J. Phys. 83(9), 863-905 (2005).

5. B. Prade, M. Franco, A. Mysyrowicz, A. Couairon, H. Buersing, B. Eberle, M. Krenz, D. Seiffer, and O. Vasseur, "Spatial mode cleaning by femtosecond filamentation in air," Opt. Lett. 31(17), 2601-2603 (2006).

6. C. P. Hauri, W. Kornelis, F. W. Helbing, A. Heinrich, A. Couairon, A. Mysyrowicz, J. Biegert, and U. Keller, "Generation of intense, carrier-envelope phase-locked few-cycle laser pulses through filamentation," Appl. Phys. B 79(6), 673-677 (2004).

7. M. Durand, A. Jarnac, A. Houard, Y. Liu, S. Grabielle, N. Forget, A. Durécu, A. Couairon, and A. Mysyrowicz, "Self-guided propagation of ultrashort laser pulses in the anomalous dispersion region of transparent solids: a new regime of filamentation," Phys. Rev. Lett. 110(11), 115003 (2013).

8. R. R. Alfano and S. L. Shapiro, "Observation of self-phase modulation and small-scale filaments in crystals and glasses," Phys. Rev. Lett. 24, 592-594 (1970).

9. M. Durand, K. Lim, V. Jukna, E. McKee, M. Baudelet, A. Houard, M. Richardson, A. Mysyrowicz, and A. Couairon, "Blueshifted continuum peaks from filamentation in the anomalous dispersion regime," Phys. Rev. A 87, 043820 (2013).

10. S. A. Hosseini, Q. Luo, B. Ferland, W. Liu, N. Akozbek, G. Roy, and S. L. Chin, "Effective length of filaments measurement using backscattered fluorescence from nitrogen molecules," Appl. Phys. B 77, 697-702 (2003).

11. C. D'Amico, A. Houard, M. Franco, B. Prade, A. Mysyrowicz, A. Couairon, and V. T. Tikhonchuk, "Conical forward THz emission from femtosecond-laser-beam filamentation in air," Phys. Rev. Lett. 98(23), 235002 (2007).

12. J. Kasparian, R. Ackermann, Y.-B. André, G. Méchain, G. Méjean, B. Prade, P. Rohwetter, E. Salmon, K. Stelmaszczyk, J. Yu, A. Mysyrowicz, R. Sauerbrey, L. Wöste, and J.-P. Wolf, "Electric events synchronized with laser filaments in thunderclouds," Opt. Express 16(8), 5757-5763 (2008).

13. B. Forestier, A. Houard, I. Revel, M. Durand, Y.-B. André, B. Prade, A. Jarnac, J. Carbonnel, M. Le Nevé, J. C. de Miscault, B. Esmiller, D. Chapuis, and A. Mysyrowicz, "Triggering, guiding and deviation of long air spark discharges with femtosecond laser filament," AIP Adv. 2(1), 012151 (2012).

14. Z. A. Kudyshev, M. C. Richardson, and N. M. Litchinitser, "Virtual hyperbolic metamaterials for manipulating radar signals in air," Nat. Commun. 4, 2557 (2013).

15. Y. Liu, M. Durand, S. Chen, A. Houard, B. Prade, B. Forestier, and A. Mysyrowicz, "Energy exchange between femtosecond laser filaments in air," Phys. Rev. Lett. 105(5), 055003 (2010).

16. J. Liu, W. Li, H. Pan, and H. Zeng, "Two-dimensional plasma grating by non-collinear femtosecond filament interaction in air," Appl. Phys. Lett. 99(15), 151105 (2011). 
17. X. Yang, J. Wu, Y. Peng, Y. Tong, P. Lu, L. Ding, Z. Xu, and H. Zeng, "Plasma waveguide array induced by filament interaction," Opt. Lett. 34(24), 3806-3808 (2009).

18. A. Brodeur and S. L. Chin, "Band-gap dependence of the ultrafast white-light continuum," Phys. Rev. Lett. 80(20), 4406-4409 (1998).

19. A. Brodeur and S. L. Chin, "Ultrafast white-light continuum generation and self-focusing in transparent media," J. Opt. Soc. Am. B 16(4), 637-650 (1999).

20. C. Nagura, A. Suda, H. Kawano, M. Obara, and K. Midorikawa, "Generation and characterization of ultrafast white-light continuum in condensed media," Appl. Opt. 41(18), 3735-3742 (2002).

21. S. Tzortzakis, B. Lamouroux, A. Chiron, M. Franco, B. Prade, A. Mysyrowicz, and S. D. Moustaizis, "Nonlinear propagation of subpicosecond ultraviolet laser pulses in air," Opt. Lett. 25(17), 1270-1272 (2000).

22. A. A. Said, M. Sheik-Bahae, D. J. Hagan, T. H. Wei, J. Wang, J. Young, and E. W. Van Stryland, "Determination of bound-electronic and free-carrier nonlinearities in ZnSe, GaAs, CdTe, and ZnTe," J. Opt. Soc. Am. B 9(3), 405-414 (1992).

23. H. Wenisch, K. Schüll, D. Hommel, G. Landwehr, D. Siche, and H. Hartmann, "Molecular beam epitaxial growth and characterization of $\mathrm{ZnSe}$ on (001) ZnSe substrates and its application in light-emitting diodes," Semicond. Sci. Technol. 11(1), 107-115 (1996).

24. Handbook of Optics, 3rd ed. (McGraw-Hill, 2009), Vol. 4.

25. V. Y. Degoda and A. O. Sofienko, "Specific features of the luminescence and conductivity of zinc selenide on exposure to X-ray and optical excitation," Semiconductors 44(5), 568-574 (2010).

26. M. V. Nazarov, "Cathodoluminescencedefectoscopy of ZnS and ZnSe crystals," Mater. Sci. Eng. B 91-92, 349$352(2002)$.

27. F. Théberge, W. Liu, P. T. Simard, A. Becker, and S. L. Chin, "Plasma density inside a femtosecond laser filament in air: Strong dependence on external focusing," Phys. Rev. E Stat. Nonlinear Soft Matter Phys. 74(3), 036406 (2006)

\section{Introduction}

Propagation of powerful femtosecond laser beams in transparent materials can give rise to a non-linear regime called filamentation. When a laser pulse with a peak power exceeding the critical power for self-focusing $\left(\mathrm{P}_{\mathrm{cr}}=100 \mathrm{~kW}\right.$ at $1.4 \mu \mathrm{m}$ in $\left.\mathrm{ZnSe}[1,2]\right)$ propagates, a strong Kerr effect will modify the index of the medium and induce self-focusing. As the laser beam self-focuses its intensity will progressively rise to the point where the medium becomes ionized by multiphoton ionization. The free electrons generated contribute negatively to the refractive index, which will lead to the defocusing of the beam. The competition between diffraction, dispersion, self-focusing, plasma defocusing, and absorption will result in a propagation regime where the beam size stays constant and where a thin plasma channel is generated $[3,4]$. During filamentation, several non-linear phenomena affect the pulse, such as self-cleaning [5], pulse compression [6,7] and white-light continuum generation [8,9]. The Kerr effect also affects the temporal evolution of the pulse, giving rise to continuum generation through self-phase modulation [8]. Fluorescence emission resulting from the plasma and spectral broadening are two characteristic phenomena that can be used to identify filamentation $[3,10]$. Numerous applications of filamentation have been proposed and several of them, such as $\mathrm{THz}$ generation [11], triggering lightning [12,13] and micro-wave guiding [14], are based on the plasma generated. Moreover, non-linear interactions between two filaments have been proven to be governed by the formation of a plasma grating [15-17]. It has been proposed that laser beams with shorter wavelengths will be more efficiently absorbed when filamenting in air, and therefore generate plasma channels of greater density. However as a laser filament is maintained by a highly nonlinear and dynamic "equilibrium", it is difficult to predict how a change in the absorption regime will affect the stability of the filament.

Previous experiments have demonstrated the influence that the band gap of the material, $\mathrm{E}_{\mathrm{g}}$, has over spectral broadening by self-phase modulation. Brodeur et al. showed that, for the same laser wavelength, materials with larger bandgaps would create broader supercontinua $[18,19]$. They identified calcium fluoride and lithium fluoride as the best materials for obtaining broad continua. Nagura et al. proposed in 2002 that this dependence was due to the competition between self-phase modulation and two-photon absorption [20]. They also deduced a threshold for continuum generation expressed by the relation $E_{g} / h v_{0}=2$, where 
$h v_{0}$ corresponded to the photon energy. Since the generation of supercontinuum and the formation of laser filaments are governed by similar physical processes, the material bandgap or ionization energy and the competition between self-phase modulation and multi-photon absorption can be expected to have a significant impact on filament formation as well. Couairon et al. have suggested that for filamentation in gases, if the ionisation potential is too low, $U_{i}<3 h v_{0}$, the attenuation of the pulse by two- or three-photon absorption will be sufficiently large to inhibit the formation of filaments [3]. When the number of photon required for multi-photon ionization decreases, a greater number of free electrons is produced [21]. Non-linear absorption is therefore greater, and can exert a much greater influence on the filamentation process. However, a direct verification of this threshold without depending on spectral broadening measurements has not been demonstrated.

In this letter, we describe a visual verification of the $E_{g} / h v_{0}=2$ threshold, as well as observations of how different multi-photon absorption regimes affect filament formation. We explore this threshold by progressively changing the input wavelength used for laser filamentation in a Zinc Selenide (ZnSe) crystal. By shifting the wavelength from $800 \mathrm{~nm}$ $\left(h v_{0}=1.55 \mathrm{eV}\right)$ to $2.4 \mu \mathrm{m}\left(h v_{0}=0.52 \mathrm{eV}\right)$ we explore the range of multi-photon ionization from two-photon to six-photon utilizing the fact that, $E_{g Z n S e}=2.67 \mathrm{eV}[22,23]$. The use of $\mathrm{ZnSe}$ is very convenient since the dispersion regime does not change over this wavelength range [24]. The influence of the number of photons needed for ionization during filamentation can therefore be studied without having to take into account the influence of the dispersion regime [7,9]. Figure 1 shows the evolution of the dispersion, as well as the linear and nonlinear indices of $\mathrm{ZnSe}$ over the wavelength range of interest.

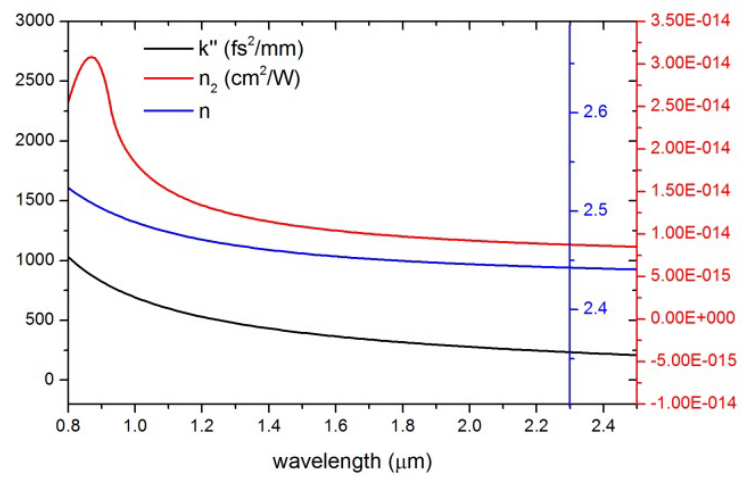

Fig. 1. Dispersion, refractive index and non-linear refractive index of $\mathrm{ZnSe}[2,22,23]$.

\section{Experimental set-up}

In this experiment we used a kilohertz CPA laser system delivering pulses of $0.5 \mathrm{~mJ}, 50 \mathrm{fs}$ at $800 \mathrm{~nm}$ to pump a commercial Optical Parametric Amplifier (OPA) system "Palitra" from Quantronix. This OPA can deliver pulses with wavelength tunable from $0.4 \mu \mathrm{m}$ to $2.5 \mu \mathrm{m}$ and with a pulse duration of $\sim 30$ fs. To study the evolution of filamentation with respect to the number of photons necessary for ionization, we set the OPA at wavelengths in the band from $800 \mathrm{~nm}$ to $2.4 \mu \mathrm{m}$, which corresponds to the multi-photon absorption regime of between two to six photons. For all the wavelengths considered, the energy of the pulses (between $6 \mu \mathrm{J}$ to $20 \mu \mathrm{J})$ was such that the input pulse power was well above the critical power for filamentation in $\mathrm{ZnSe}(\sim 70 \mathrm{~kW}$ at $1.2 \mu \mathrm{m}$ and $\sim 400 \mathrm{~kW}$ at $2.4 \mu \mathrm{m})$. In each case the pulse was focused with a $20 \mathrm{~cm}$ focal length lens on the front face of the sample. A digital camera coupled with a macroscopic objective was used to visualize the filament (see Fig. 2). The continuum generated by filamentation was focused on a diffusive white screen and measured by two 
spectrometers (HR 4000 and NIRQUEST from Ocean Optics) covering the spectral range 300 $\mathrm{nm}-2.5 \mu \mathrm{m}$.

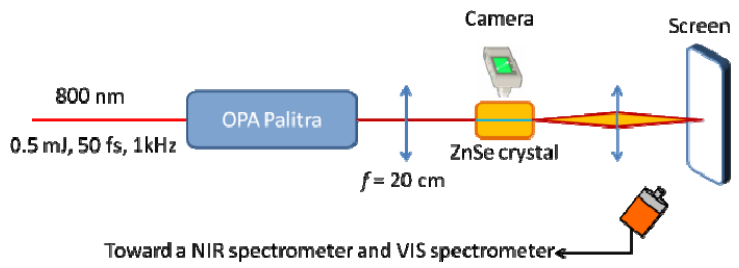

Fig. 2. Experimental set-up.

\section{Results}

Firstly, filamentation at $800 \mathrm{~nm}$ was examined and, as expected [3,18-20], two-photon absorption was strong enough to prohibit filamentation. As shown in Fig. 3, the pulse spectrum exhibits no significant broadening. Moreover a bright blue spot can be observed on the front face of the crystal (insert on Fig. 3) as a result of two-photon photoluminescence. With the laser source tuned to $600 \mathrm{~nm}$ the same result was obtained. Filamentation does not occur if the wavelength used does not satisfy the criteria: $h v_{0}<E_{g} / 2$ since in this case strong two-photon absorption is the dominant non-linear phenomena.

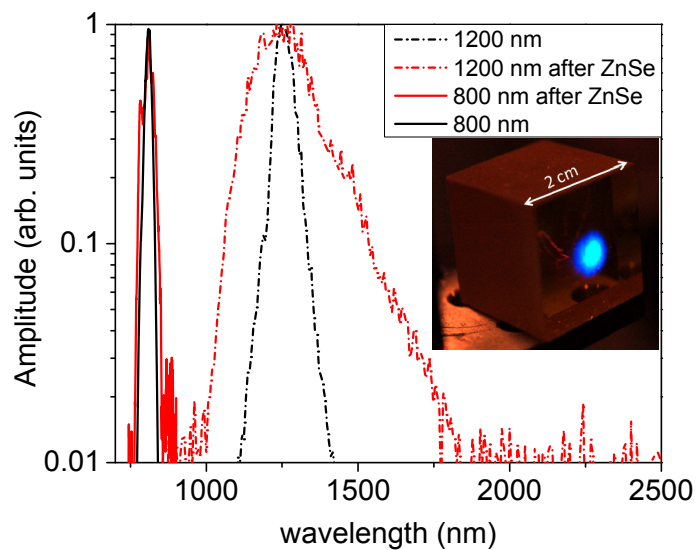

Fig. 3. Normalized laser spectrum before and after propagation/filamentation in the ZnSe crystal for $\lambda_{0}=800 \mathrm{~nm}$ and $\lambda_{0}=1200 \mathrm{~nm}$. In insert: Photography of the front face of the crystal, illustrating the blue light resulting from two photons absorption for $\lambda_{0}=800 \mathrm{~nm}$.

Plasma emission from a pulse filamenting in gas is often used to monitor filamentation as it indicates the region where the femtosecond pulse reaches high intensities. In a similar manner, photoluminescence is expected to be a conveniently observable proxy of filamentation in solids. The photoluminescence created inside the bulk $\mathrm{ZnSe}$ by the filamenting pulse was first characterized for four different initial wavelengths. The spectrum of the photoluminescence collected by a $45 \mathrm{~mm}$ focal length lens perpendicular to the axis of pulse propagation, was focused into a $0.5 \mathrm{~m}$ Czerny-Turner spectrometer (2500i, Princeton Instruments) with 600 line $/ \mathrm{mm}$ grating coupled to a 256 x 1024 ICCD camera (iStar 720, Andor). As shown in Fig. 4, the side emission observed from the $\mathrm{ZnSe}$ is independent of the input laser wavelength and corresponds to the edge emission of $\mathrm{ZnSe}$ at the bandgap energy of $2.67 \mathrm{eV}$, where the measured central wavelength is $\sim 460 \mathrm{~nm}[25,26]$. This confirms that 
the emission can be used to monitor the high intensity region of the femtosecond pulse while it propagates inside the medium.

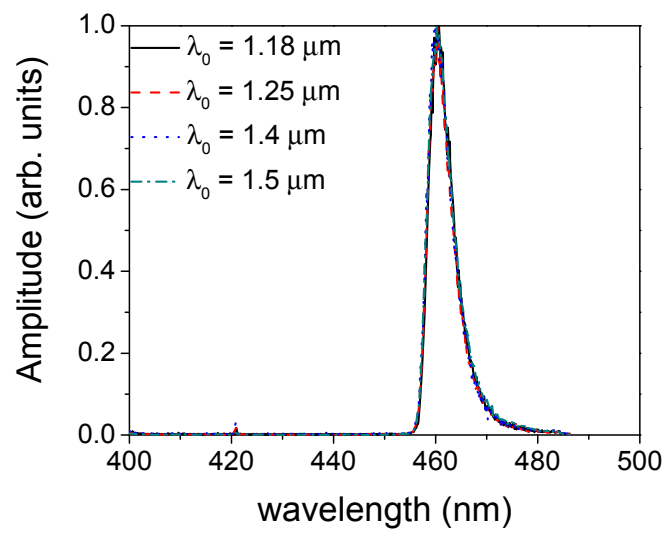

Fig. 4. Photoluminescence spectra measured from the side of the filament for different laser wavelength.

The propagation of the filamenting pulse inside the bulk material was then studied as a function of the OPA wavelength for peak powers well in excess of the critical power necessary to induce filamentation. In this case, multiple filaments are expected to appear. As the wavelength of the input pulses was progressively increased above the threshold for twophoton absorption, a bright blue string of light characteristic of filamentation became visible inside the bulk material. Figures 5(a) and 5(b) show luminescence inside the $\mathrm{ZnSe}$ for 1.15 $\mu \mathrm{m}$ and $1.2 \mu \mathrm{m}$, corresponding to the regime where three-photon ionization occurs. The strongest absorption/photoluminescence is still observed at the front face of the sample, but, in contrast to the two-photon absorption case, the absorption region penetrates the surface. For the initial part of the propagation, the photoluminescence does not exhibit a focused zone extending beyond the Rayleigh length. Théberge et al. [27] have demonstrated that geometrical focusing can lead to greater plasma densities in filaments. In the 3-photon absorption case, our observations suggest that the increased absorption and plasma generation due to focusing is significant enough to arrest filamentation. Nonetheless, self-focusing takes over once absorption becomes weaker beyond the geometrical focus and initial collapse region, and the beam undergoes refocusing and generates a string of blue emission lasting over $0.5-1 \mathrm{~cm}$, which is indicative of laser filamentation. This demonstrates that filamentation can occur in the regime of three-photon absorption, though, unlike filaments observed in more "traditional" conditions (800 $\mathrm{nm}$ pulses in gases, water or large bandgap solids), the filamentation zone is detached from the geometrical focus.

Figures 5(c) and 5(d) show the results for $1.4 \mu \mathrm{m}$ and $1.7 \mu \mathrm{m}$, corresponding to the fourphoton ionization regime. In the case of $1.4 \mu \mathrm{m}$, the absorption around the geometrical focus is still strong enough to prevent filamentation from occurring. Due to the broad spectrum of the $1.4 \mu \mathrm{m}$ pulse, a significant amount of its energy contributes to three-photon instead of four-photon absorption (transition at $1.393 \mu \mathrm{m}$ ). It is therefore not surprising that characteristics of the three-photon absorption regime are observed. On the other hand, the spectrum of the $1.7 \mu \mathrm{m}$ pulse is well above this three- and four-photon absorption transition, and the absorption near the front face of the ZnSe crystal is insufficient to arrest self-focusing and the formation of filaments. As predicted by Couairon et al., once the three-photon absorption barrier has been overcome, the intense short pulse can undergo filamentation without being initially perturbed by the multi-photon absorption process, even if absorption can be very significant in the collapse region [3]. 
Once the initial input wavelength used for filamentation reaches five- and six-photon ionization regime, as it is the case for $1.9 \mu \mathrm{m}, 2 \mu \mathrm{m}$ and $2.4 \mu \mathrm{m}$ initial wavelength (respectively Figs. 5(e)-5(g)), absorption is significantly weaker in the initial collapse region and the filaments present better spatial stability - they form upon the initial collapse and the filament bunch is continuous.

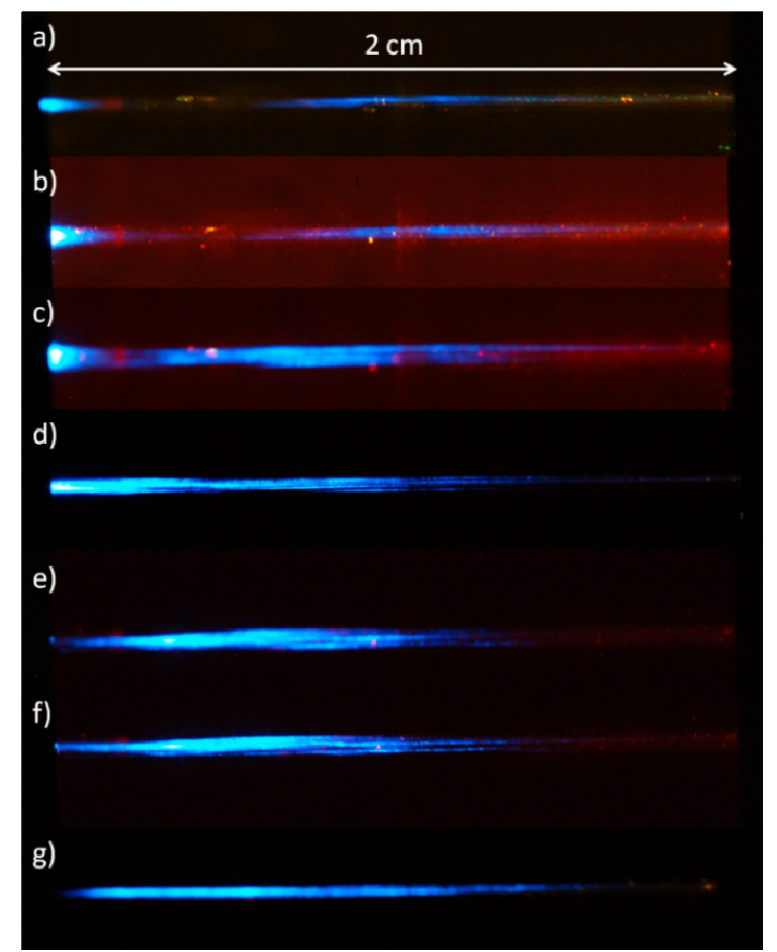

Fig. 5. Photography by the side face of the crystal bulk. One can see the photoluminescence for different wavelength (a) $1.15 \mu \mathrm{m}$ (b) $1.2 \mu \mathrm{m}$ (c) $1.4 \mu \mathrm{m}$ (d) $1.7 \mu \mathrm{m}$ (e) $1.9 \mu \mathrm{m}$ (f) $2 \mu \mathrm{m}$ (g) 2.4 $\mu \mathrm{m}$.

Other than photoluminescence of the medium, spectral broadening of the transmitted laser pulse was also investigated as a diagnostic of laser filamentation [3]. Spectra of the transmitted laser pulse were taken for the different laser wavelength both with the $\mathrm{ZnSe}$ crystal, and without it after linear propagation in air (see Fig. 1 for examples at $\lambda_{0}=800 \mathrm{~nm}$ and $1.2 \mu \mathrm{m}$ ). The broadening $\Delta \omega$ resulting from filamentation was then calculated from spectra measurement before and after filamentation (Fig. 6). In the two-photon absorption regime, an input pulse at $800 \mathrm{~nm}$ undergoes no spectral broadening. Once the wavelength used for filamentation exceeds the two-photon threshold, (for $\lambda_{0}=1.15 \mu \mathrm{m}$ and $1.2 \mu \mathrm{m}$ ) a small broadening of $\Delta \omega \sim 100 \mathrm{~nm}$ is observed. For the four-photon regime the broadening becomes larger $\Delta \omega \sim 300 \mathrm{~nm}$. The special case of $\lambda_{0}=1.4 \mu \mathrm{m}$ exhibits an intermediate amount of broadening explainable by the transitional behavior between the two regimes. Finally, for the five-photon ionization regime, the broadening is much larger at $\Delta \omega \sim 450 \mathrm{~nm}$. These results are in good agreement with the photoluminescence measurement; filaments can develop as soon as the input wavelength exceeds the two-photon absorption level. However, for more stable and stronger filamentation features, including spatial stability and spectrum broadening, the input wavelength should exceed the three-photon absorption limit of the material. 


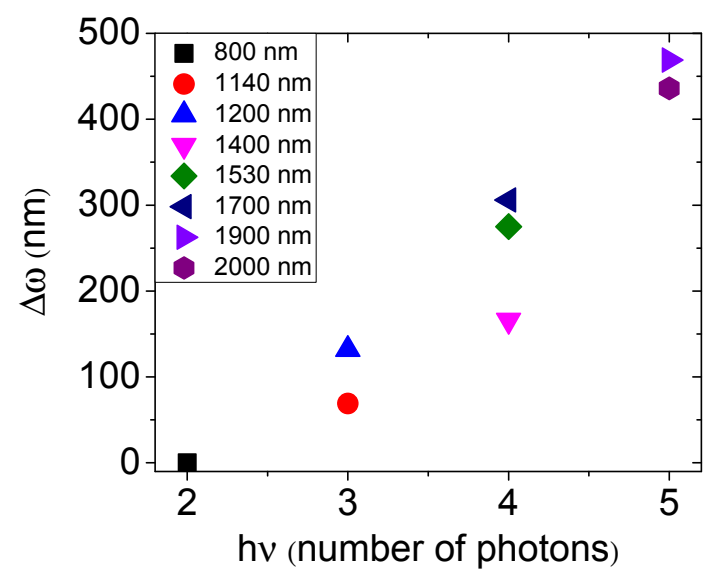

Fig. 6. Spectral broadening $\Delta \omega=\Delta \omega_{\text {filament }}-\Delta \omega_{0}$ as a function of number of photons needed for ionization, for each initial wavelength used for filamentation.

\section{Conclusion}

In conclusion, we have directly observed the formation of filaments as a function of the input wavelength, while staying in the same dispersion regime of a material, to assess the detrimental influence of two- and three-photon ionization on filamentation. For this purpose we used a $\mathrm{ZnSe}$ crystal, as its bandgap $E_{\text {gZnSe }}=2.67 \mathrm{eV}$ allowed us to study the regimes from two-photon ionization to six-photon ionization using a commercial OPA tuneable from 600 $\mathrm{nm}$ to $2.5 \mu \mathrm{m}$. The evolution of both photoluminescence emission and spectral broadening showed that filamentation occurred as soon as the multi-photon ionization involved at least three photons. We can therefore conclude that for filamentation to occur, the condition $h v_{0}<E_{g} / 2$ must first be achieved. Furthermore, when three-photon absorption is dominant, features indicative of filamentation begin to be observable, but the process remains easily perturbed by external conditions (e.g. external focusing) that changes the balance between Kerr-induced self-focusing and the opposing defocusing and absorption effects. As we move on to higher order multi-photon ionization regimes, filaments with greater spatial stability and spectral broadening can be obtain as the absorption at the initial collapse is no longer strong enough to arrest the filamentation process. Depending on the application and the given material (or wavelength), the wavelength (or material) can therefore be adjusted to optimize for the desired filament properties, such as spectral broadening, plasma density, and longitudinal stability.

\section{Acknowledgments}

This work was funded by the Army Research Office / Multidisciplinary University Research Initiative "Light Filamentation Science" Contract \#W911NF1110297 and the State of Florida. 